\title{
MESHFREE MODELLING OF DYNAMIC FRACTURE IN HIGH-STRENGTH CONCRETE
}

\author{
PEDRO NAVAS ${ }^{\dagger}$, BO LI ${ }^{\ddagger}$ AND RENA C. YU* \\ $\dagger$ University of Castilla-La Mancha \\ Ciudad Real, Spain \\ e-mail: pedro.navas@uclm.es \\ ${ }^{\dagger}$ Case Western Reserve University \\ Cleveland, OH USA \\ e-mail: bo.li14@case.edu \\ *University of Castilla-La Mancha \\ Ciudad Real, Spain \\ e-mail: rena@uclm.es
}

Key words: Meshfree modelling, OTM, Eigenerosion, High-strength concrete, Dynamic fracture

\begin{abstract}
The present work is concerned with the meshfree modelling of dynamic fracture in concrete. The Optimal Transportation Meshfree(OTM) method is chosen for its numerous advantages such as the exact mass transport, the satisfaction of the continuity equation, exact linear and angular momentum conservation, consequently numerical problems such as spurious modes, tensile instabilities are resolved naturally. In addition, the energy-based variational material point failure algorithm (Eigen-Erosion) is employed to predict the crack propagation in concrete under dynamic loading conditions due to the properties of $\Gamma$-Convergence and mesh independence. The OTM and EigenErosion approaches are validated in our simulations of dynamic fracture propagation in concrete loaded in the three point bending tests through a drop weight impact device. High fidelity predictions of the reaction and impact forces are obtained by comparing to the experimental measurements.
\end{abstract}

\section{INTRODUCTION}

The rate effect on fracture propagation in concrete with or without reinforcements has been the centre of interest for the last three decades, from both experimental and numerical standpoints. Within the framework of finite element methods, typical techniques to capture crack propagation vary from employing cohesive approaches [1, 2] by adaptively inserting cohesive elements [3-5] at solid element boundaries to adopting crack band theory [6] or handling arbitrary crack paths by level set representation of the fracture surface [7, 8]. Within the scenario of meshfree meth- ods, the visibility criterion [9] or particle erosion [10-13] have been opted for to reproduce fracture propagation. In the current work, we attempt to model the dynamic fracture propagation in high-strength concrete (HSC) employing Optimal Transportation Meshfree (OTM) method and the energy-based variational material point failure algorithm (EigenErosion) scheme [12-15]. Such a scheme is chosen for its numerous advantages such as the exact mass transport, the satisfaction of the continuity equation, exact linear and angular momentum conservation. Since the deformation and velocity fields are interpolated from nodal values 
using max-ent shape functions, the Kroneckerdelta property at the boundary makes it possible for the direct imposition of essential boundary conditions. Fracture is modelled through material-point failure, i.e. each material point can be either intact or be completely failed and has no load bearing capacity. The crack set is approximated by means of eigen-deformations, which enable the material to develop displacement jumps at no cost of local elastic energy. The feasibility of such methodology has been demonstrated for brittle materials [11,12] and dynamic fragmentation of metals [13].

In 1980s, using high speed photography, Mindess and Bentur [16] carried out studies of the fracture, under impact loading, of plain concrete, fibre reinforced concrete, and concrete with conventional reinforcement. They concluded that the cracking process under impact loading is not substantially different from that which occurs under static loading, even though the absorption of energy is quantitatively different due to the inertia effect. In a sequel, Mindess, Banthia and Yan [17] showed that both the fracture toughness and the fracture energy were dramatically increased under impact loading. Banthia et al. [18] also presented a dropweight impact-testing machine for generating impact flexural loading; by replacing the distributed inertia load with a generalized point inertial load and subtracting it from the tup load, they obtained the true bending load equivalent to the static case, when plotted with respect to the load-point deflection, the area below was considered as the dynamic fracture energy. This procedure, which we term the Hypothesis of Banthia et al., has been widely employed by Banthia, Mindess and co-workers [19-21], and recently by Ruiz and coworkers [22-25]. However, except the analytic demonstration by Banthia et al. [18] with significant simplification, the validity of such a procedure has not been rigorously proved. By directly modeling both the projectile and target in 3D under dynamic loading conditions, we aim to examine the validity of the Hypothesis of Banthia et al. in the real situation without making any assumptions and simplification.

Next the experimental setup and observations are presented. The meshfree methodology and eigen-erosion algorithm for fracture are summarised in Section 3. Numerical results and discussion are given in Section 4. Finally, relevant conclusions are drawn in Section 5.

\section{EXPERIMENTAL OBSERVATIONS}

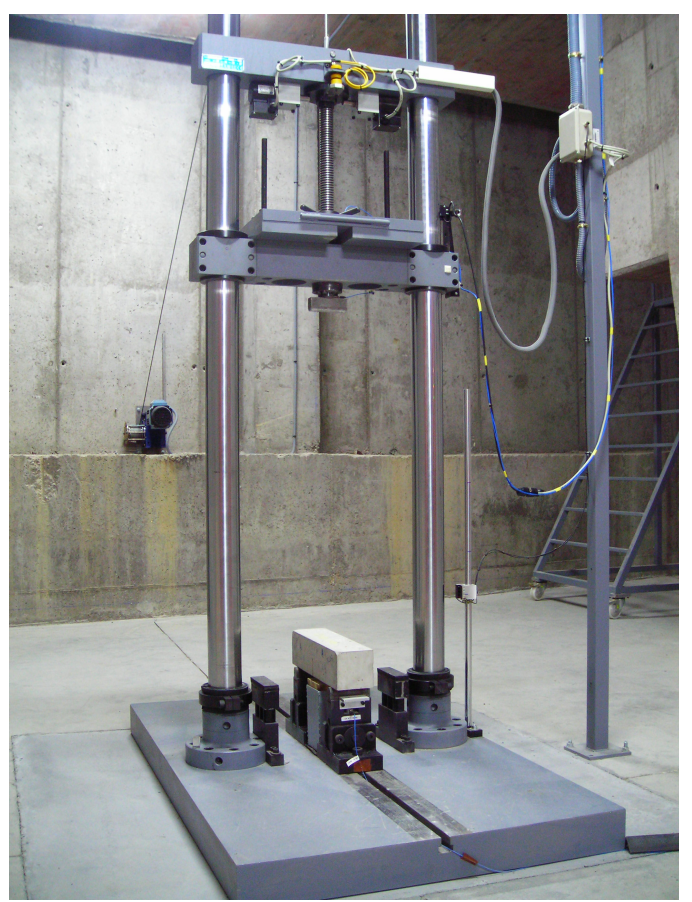

Figure 1: The drop-weight machine designed at the Laboratory of Materials and Structures, University of CastillaLa Mancha.

In order to study the loading-rate effect in concrete, a drop-weight impact instrument [25], see Fig. 1. was designed and constructed in the Laboratory of Materials and Structures at the University of Castilla-La Mancha. Three-point bending tests on notched beams were conducted under impact loading. It has the capacity to drop a $316 \mathrm{~kg}$ mass from heights of up to $2.6 \mathrm{~m}$, and can accommodate flexural specimens with spans of up to approximately $1.6 \mathrm{~m}$. In this study, an impact hammer of $120.6 \mathrm{~kg}$ was employed to drop from the height of $360 \mathrm{~mm}$ with the corresponding impact speed of $2640 \mathrm{~mm} / \mathrm{s}$. Three specimens were tested for this impact speed. The impact force is measured by a piezo- 
electric force sensor. In addition, the reaction force is determined by two force sensors located between the support and the specimen. A strain amplifier and two oscilloscopes were used to acquire the data from the strain gauges, located as it can be seen in Fig. 2,

The beam dimensions were $100 \mathrm{~mm} \times 100 \mathrm{~mm}$ $(B \times D)$ in cross section, and $420 \mathrm{~mm}$ in total length, L. The initial notch-depth ratio was approximately 0.5 , and the span, $\mathrm{S}$, was fixed at $300 \mathrm{~mm}$ during the tests, see Fig. 2. Each specimen was removed from the moist room one day before the test and restored to the chamber after bonding the strain gauges. The specimen surface was polished and all four strain gauges were bonded to that surface, with a distance of $10 \mathrm{~mm}$ between each neighbouring gauge. Since a running crack in concrete is often deflected by aggregates along its path, the four strain gauges were bonded $10 \mathrm{~mm}$ apart from the centerline of the beam, see Fig. 2. Those strain gauges provided not only the strain history at the bonded positions, but also the time at which the crack tip reaches each strain gauge. For more information see [23].

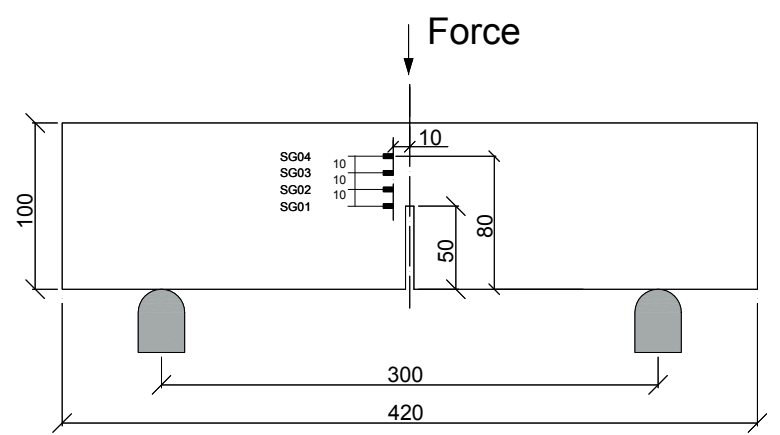

Figure 2: Geometry and dimension of the concrete beam (in $\mathrm{mm}$ ) and location of the strain gauges.

The material was characterised with independent tests and the measured material properties, such as the compressive strength, $f_{c}$, the tensile strength, $f_{t}$, the specific fracture energy, $G_{F}$ and the elastic modulus, $E$, are given in Table 2 .
Table 1: Mechanical properties of the HSC tested, with the density of $2368 \mathrm{~kg} / \mathrm{m}^{3}$.

\begin{tabular}{c|cccc}
\hline & $f_{c}$ & $f_{t}$ & $G_{F}$ & $E$ \\
& $(\mathrm{MPa})$ & $(\mathrm{MPa})$ & $(\mathrm{N} / \mathrm{m})$ & $(\mathrm{GPa})$ \\
\hline Mean & 102.7 & 5.4 & 141 & 31 \\
Std. Dev & 2 & 0.8 & 9 & 2 \\
\hline
\end{tabular}

\section{THE NUMERICAL METHODOLOGY}

For completeness, we summarise the basics of the OTM scheme and the eigen-erosion algorithm to treat fracture within the meshfree framework.

\subsection{The OTM scheme}

In this work the OTM method [14] is employed in order to fully discretise the problem in space and time. It is a meshfree updated Lagrangian numerical scheme that combines concepts from Optimal Transportation theory local max-ent meshfree approximation and material point sampling [26]. The method has been used in applications involving dynamic deformation and failure of materials with demonstrated excellent results. The numerous advantages mentioned before ensure its robustness and stability in numerical calculations.

The local max-ent approximation scheme defined by Arroyo and Ortiz [26] is employed for fields requiring differentiation, such as deformation and velocity fields. The employed local max-ent function (LME), as a Pareto set, is optimal for $\beta \in(0, \infty)$. It is obtained as:

$$
N_{a}(\mathbf{x})=\frac{\exp \left[-\beta\left|\mathbf{x}-\mathbf{x}_{\mathbf{a}}\right|^{2}+\boldsymbol{\lambda}^{*} \cdot\left(\mathbf{x}-\mathbf{x}_{\mathbf{a}}\right)\right]}{Z\left(\mathbf{x}, \boldsymbol{\lambda}^{*}(\mathbf{x})\right)},
$$

where

$Z(\mathbf{x}, \boldsymbol{\lambda})=\sum_{a=1}^{n} \exp \left[-\beta\left|\mathbf{x}-\mathbf{x}_{\mathbf{a}}\right|^{2}+\boldsymbol{\lambda} \cdot\left(\mathbf{x}-\mathbf{x}_{\mathbf{a}}\right)\right]$,

being $\lambda^{*}(\mathbf{x})$ the unique minimizer for $\log Z(\mathbf{x}, \boldsymbol{\lambda})$. The parameter $\beta$ is related with the discretization size (or nodal spacing), $h$, and the constant, $\gamma$, which controls the locality of the shape functions, as follows 


$$
\beta=\frac{\gamma}{h^{2}}
$$

For a uniform nodal spacing, $\beta$ is also a constant, thus the first derivatives of the LME shape functions can be obtained by employing the following expression:

$$
\nabla N_{a}^{*}=-N_{a}^{*}\left(\mathbf{J}^{*}\right)^{-1}\left(\mathbf{x}-\mathbf{x}_{\mathbf{a}}\right),
$$

where $\mathbf{J}$ is the Hessian matrix, defined by:

$$
\begin{aligned}
\mathbf{J}(\mathbf{x}, \boldsymbol{\lambda}, \beta) & =\frac{\partial \mathbf{r}}{\partial \boldsymbol{\lambda}} \\
\mathbf{r}(\mathbf{x}, \boldsymbol{\lambda}, \beta) & \equiv \partial_{\boldsymbol{\lambda}} \log Z(\mathbf{x}, \boldsymbol{\lambda}) \\
& =\sum_{a} N_{a}(\mathbf{x}, \boldsymbol{\lambda}, \beta)\left(\mathbf{x}-\mathbf{x}_{\mathbf{a}}\right)(6)
\end{aligned}
$$

Note that the objective of the above procedure is to find the $\boldsymbol{\lambda}$ which minimizes $\log Z(\mathbf{x}, \boldsymbol{\lambda})$. This unconstrained minimization problem with a strictly convex objective function can be solved efficiently and robustly by a combination of the Newton-Raphson method and NelderMead Simplex algorithm [26, 27].

Two sets of points, namely, nodal points and material points are defined. In calculations, the max-ent shape functions are reconstructed continuously from the nodal set, which changes in every step by adapting to the displacements of the nodes. This overcomes the essential difficulties that arise in fixed grid-based numerical schemes like Lagrangian and Eulerian finite element methods. Material points result from the spatial approximation of the mass densities $\rho_{h, k}(x)$ by $M$ mass points

$$
\rho_{h, k}(x)=\sum_{p=1}^{M} m_{p} \delta\left(x-x_{p, k}\right)
$$

where $x_{p, k}$ represents the position at time $t_{k}$ of material point $p, m_{p}$ is the mass of the material point and $\delta\left(x-x_{p, k}\right)$ is the Dirac-delta distribution centred at $x_{p, k}$. Material points designate fix material points of the body, are convected by the deformation, carry a fixed mass, serve the purpose of integration points for the calculation of the effective nodal forces and masses, and store all local state data. The spatial discretisation is completed by approximating the deformation mapping as

$$
\varphi_{h, k \rightarrow k+1}(x)=\sum_{a=1}^{N} x_{a, k+1} N_{a, k}(x)
$$

where $x_{a, k+1}$ is the position at time $t_{k+1}$ of node $a$, and $N_{a, k}(x)$ are max-ent shape functions defined over the configuration at time $t_{k}$. The interpolation at a material point $x_{p, k}$ depends only on the nodes contained in a small local neighbourhood of the material point. This reconstruction of the local neighbourhoods leads to a new reconnection of the material points and the nodal set.

\subsection{Eigenerosion algorithm}

Within the context of OTM formulation, fracture can be modelled simply by failing material points according to an energy-release criterion. When the material points are failed, they are neglected from the computation of stresses in the model, which approximates the presence of cracks, this is the so-called eigen-erosion algorithm developed by Pandolfi et al. [11]. Next we compute the energy-release rate attendant to the failure of material point $p$ as seen in $[10-12,15]$.

$$
\begin{aligned}
G_{p, k+1} & =\frac{C \epsilon}{m_{p, k+1}} \sum_{x_{q, k+1} \in B_{\epsilon}\left(x_{p, k+1}\right)} m_{q} W_{k}\left(F_{q, k+1}\right) \\
m_{p, k+1} & =\sum_{x_{q, k+1} \in B_{\epsilon}\left(x_{p, k+1}\right)} m_{q}
\end{aligned}
$$

where $B_{\epsilon}\left(x_{p, k+1}\right)$ is the sphere of radius $\epsilon$ centred at $x_{p, k+1}$ known as the $\epsilon$-neighbourhood of the material point, $m_{p, k+1}$ is the mass of the neighbourhood, $W_{k}\left(F_{q, k+1}\right)$ is the incremental free-energy density per unit mass and $\mathrm{C}$ is a normalising constant. A scheme of the configuration of the $\epsilon$-neighbourhood is plotted in Fig 3 .

The material point is failed when $G_{p, k+1} \geq$ $G_{c}$, where $G_{c}$ is a critical energy release rate that measures the material-specific energy, $G_{F}$ in Table 2, required to create a fracture surface of unit area. In calculations, the failed material-point set is updated at every time step 
according to this criterion. For linear elasticity, Schmidt et al. [10] have shown that this approximation converges to Griffith fracture in the limit of an infinitely fine discretisation. In order to minimise the computational effort, employing an averaging over an intermediate $\epsilon$ neighbourhood to compute the energy-release rate attendant to material-point failure, Eq. (9), is essential to convergence. The local neighbourhood averaging of the energy has the effect of eliminating spurious mesh-dependent artefacts. Indeed, erosion schemes that estimate the energy-release rate based on the energy of a single material point suffer from mesh-dependency and may overestimate the toughness of the material.

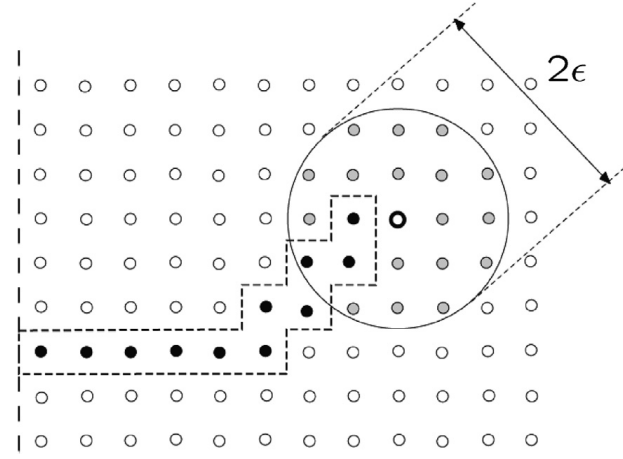

Figure 3: Scheme of a crack (black dots) as set of failed material points, and of the $\epsilon$-neighborhood (grey dots) of the material point located at the crack tip (hollow dot).

It needs to be pointed out that when a material point satisfies the erosion condition, its contribution to the internal force vector and to the material stiffness matrix is set to zero, but its contribution to the mass matrix is maintained. The mass of a material point is discarded only when an eroded material point is not connected to any nodes.

\section{NUMERICAL RESULTS AND DIS- CUSSION}

In this Section, we apply the aforementioned methodologies to simulate the dynamic fracture propagation in a three-point bend beam impacted by a drop-weight device. Both the projectile (the hammer) and the target (the concrete beam) are explicitly represented. Several levels of discretisation are employed to assess the objectiveness of the obtained results. The results obtained in this Section are from a discretisation of 16467 nodes, 81129 material points, and a nodal spacing of $2 \mathrm{~mm}$ near the middle section is maintained.

First we validate the reaction and impact forces as well as the load-line displacement against their experimental counterparts. Next the crack front evolution and strain history at the locations where the four strain gauges were bonded are extracted. Finally, the hypothesis of Banthia et al. is assessed through energy evolution.
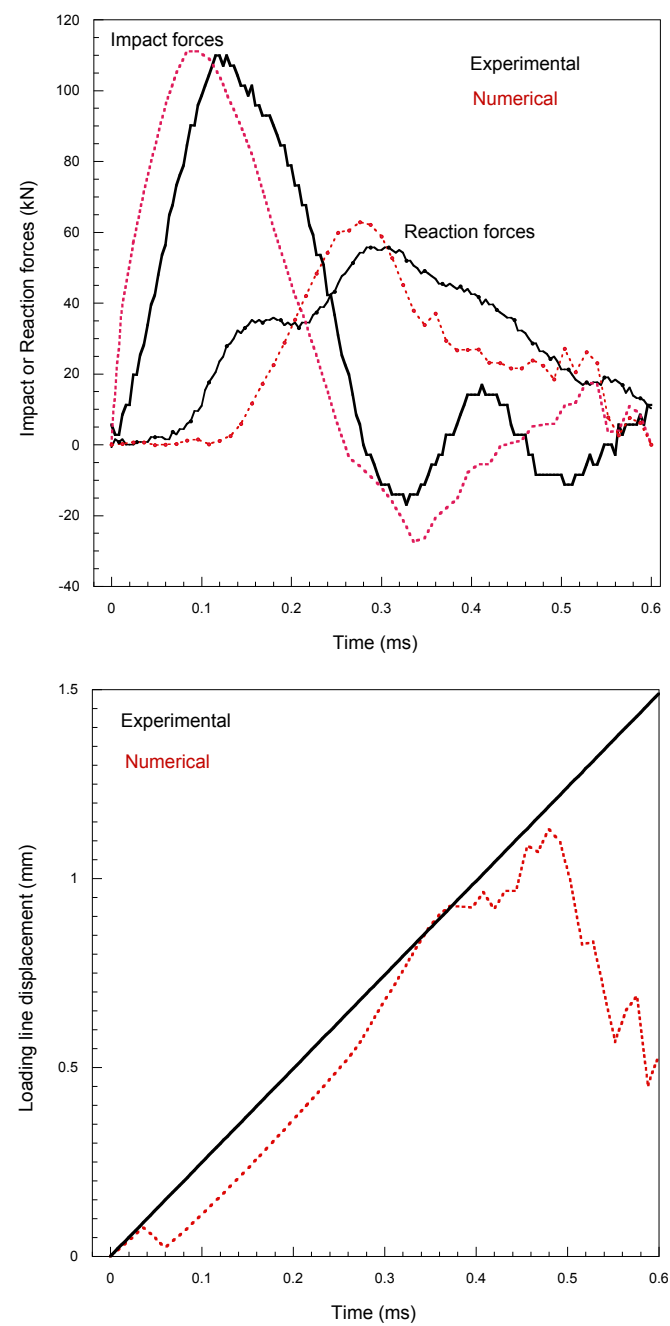

Figure 4: Comparison between experimental and computational (top) impact forces and reaction forces; (bottom) impact-line displacement. 


\subsection{Validation against experimental results}

Since the impact forces applied by the hammer and the reaction forces at the two supports were experimentally measured, they are compared with the numerical ones in Fig. 4. Note that the general trend of both forces are correctly captured. However, the time delay between the numerical and experimental forces is not physical in a sense that the curves are purposely aligned so that they start from the same reference moment.

Additionally plotted in Fig. 4 is the recorded impact-line displacement contrasted with the numerical one. The initial bump of the numerical curve can be attributed to the first contact between the hammer and the target. It is noteworthy that, when the reaction force attained its peak value at $0.28 \mathrm{~ms}$, the loadingline displacement augmented in a more steep fashion. This indicates that due to the advancement of the main crack (as can be seen in Fig. 5 . the crack front had extended from $50 \mathrm{~mm}$ to $90 \mathrm{~mm}$ at $0.26 \mathrm{~ms}$ ), the stiffness had dropped considerably. Another interesting phenomenon is at time $0.48 \mathrm{~ms}$, the loading-line displacement started to decrease. This means that the top surface of the beam had actually bounced back.

\subsection{Dynamic crack propagation}

Due to the explicit nature of our simulation, the crack front are well indicated by the newly eroded material points. The highest most recently eroded material points is extracted and plotted against time in Fig. 5. Note that the crack initiated at about $0.07 \mathrm{~ms}$ after the impact. At the beginning of the crack growth, the crack propagation velocity is about $400 \mathrm{~m} / \mathrm{s}$, then it gradually drops to around $100 \mathrm{~m} / \mathrm{s}$, until the beam is completely broken. These values and the decreasing trend agree very well with the experimentally measured ones given by Zhang et al. [23].

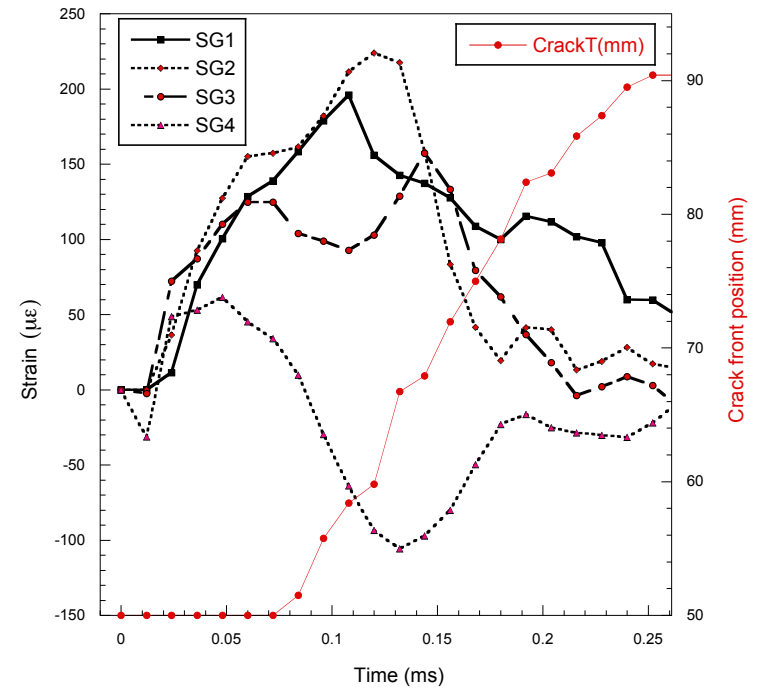

Figure 5: Numerically obtained strain evolution at the same locations where the four strain gauges are bonded and the crack-front position with respect to time.

The strain histories at the same locations where the four strain gauges were bonded are also extracted and plotted in Fig. 5. It is observed that the strain peaks are reached slightly later than the crack front. This is due to, on the one hand, the 10-mm distance between the strain gauges and the main crack surface; on the other hand, the detected crack tip might be inside, whereas the strain gauges only give the information at the surface.

\subsection{Energy evolution: the hypothesis of Banthia}

In order to characterise the fracture properties of a brittle material, the thee-point-bend configuration is often used to measure the specific fracture energy, $G_{F}$. Since the beam is in equilibrium, the measured reaction force is employed as the loading force to obtain the area below the load-deflection curve. When the load is dynamic, however, the contribution of inertia forces makes it difficult in the lab to determine the bending force which actually fractures the beam. Banthia et al. [18] proposed the scheme given in Fig. 6 to offset the influence of inertia, where in an equivalent pseudostatic state, a generalised bending load, $P_{b}$, is considered as the reaction forces at the two sup- 
ports. Consequently, the shaded area is considered as the fracture energy expenditure under dynamic loading conditions. The numerical and experimental comparison of this energy is plotted in Fig. 7. Note that the numerical curve follows the general trend of the experimental one, though the difference between these two needs further investigation. In particular, the evolution of kinetic energy, elastic energy dissipation in eroded material points will bring more insights into the understanding of the hypothesis of Banthia et al.
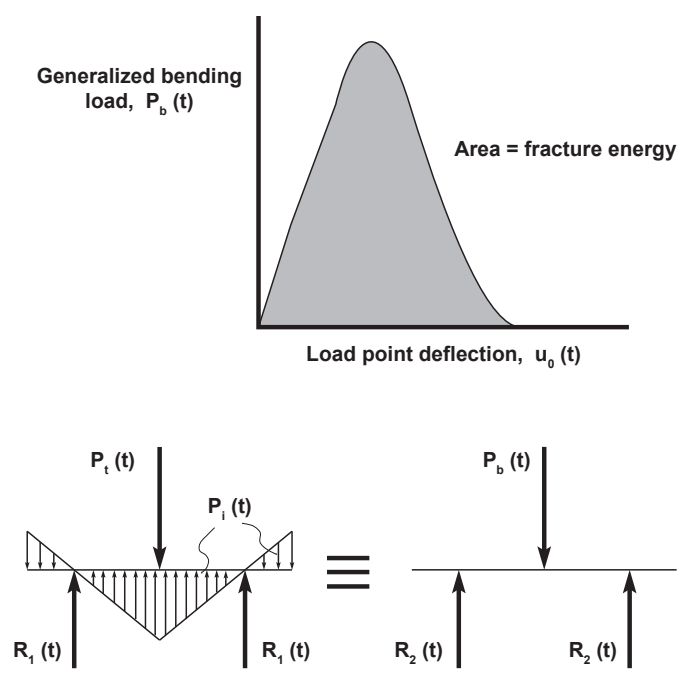

Figure 6: Scheme of calculation of the fracture energy and behaviour of the loads according to Banthia et al. [18].

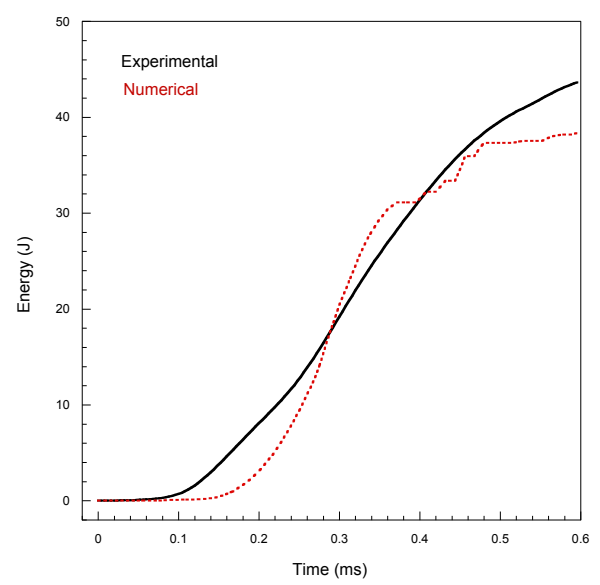

Figure 7: Comparison of the numerical and experimental energy evolution.

\section{CONCLUSIONS AND FUTURE WORK}

We have applied the Optimal Transportation Meshfree scheme and the eigen-erosion algorithm to simulate the dynamic fracture propagation in a high-strength concrete beam loaded in a three-point-bend configuration. Both the impact hammer and the beam target are explicitly discretised. The obtained impact and reaction forces as well as load-line displacements are validated against the corresponding experimental ones. The crack propagation velocity is calculated from crack-front position history, the order of magnitude compares well with that of measured through strain gauges. Further investigation is necessary in order to probe the soundness of the hypothesis of Banthia et al. The influence of strain rate on the energy release rate is also work in progress.

\section{Acknowledgements}

The authors are grateful for the discussions with Dr. Zhang, Prof. Ruiz and Dr. Cifuentes. Financial support from the Consejería de Educación, Cultura y Deportes de la Junta de Comunidades de Castilla-La Mancha, Fondo Europeo de Desarrollo Regional, under Grant No. PEII-2014-016-P, Spain, is acknowledged.

\section{REFERENCES}

[1] Barenblatt, G.L. 1962. The mathematical theory of equilibrium cracks in brittle fracture. Advances in Applied Mechanics. 7:55-129.

[2] Hillerborg, A., Modéer, M. and Petersson, P.E. 1976. Analysis of crack formation and crack growth in concrete by means of fracture mechanics and finite elements. Cement and Concrete Research. 6:773782.

[3] Ortiz, M. and Pandolfi, A. 1999. Finite-deformation irreversible cohesive elements for three-dimensional crack-propagation analysis. International Journal for Numerical Methods in Engineering 44:1267-1282 
[4] Pandolfi, A. and Ortiz, M. 2002. An Efficient Adaptive Procedure for ThreeDimensional Fragmentation Simulations. Engineering with Computers. 18(2):148159.

[5] Ruiz, G., Pandolfi, A. and M. Ortiz. 2001. Three-dimensional cohesive modeling of dynamic mixed-mode fracture. International Journal for Numerical Methods in Engineering. 52:97-120.

[6] Bažant, Z.P. and Oh, B.H. 1983. Crack band theory for fracture in concrete. $M a$ terials and Structures. 16:155-177.

[7] Belytschko, T., Chen, H., Xu, J. and Zi, G. 2003. Dynamic crack propagation based on loss of hyperbolicity and a new discontinuous enrichment. International Journal for Numerical Methods in Engineering. 58:1873-1905.

[8] Gravouil, A., Moës, N. and Belytschko, T. 2002. Non-planar 3D crack growth by the extended finite element and level sets-Part II: level set update. International Journal for Numerical Methods in Engineering. 53:2569-2586.

[9] Rabczuk, T. and Belytschko, T. 2004. Cracking particles: a simplified meshfree method for arbitrary evolving cracks. International Journal for Numerical Methods in Engineering. 61:2316-2343.

[10] Schmidt, B., Fraternali, F. and Ortiz, M. 2009. Eigenfracture: an eigendeformation approach to variational fracture. SIAM J. Multiscale Model. Simul. 7:1237-1266.

[11] Pandolfi, A. and Ortiz, M. 2012. An eigenerosion approach to brittle fracture. International Journal for Numerical Methods in Engineering 92:694-714.

[12] Pandolfi, A., Li, B. and Ortiz, M. 2013. Modeling fracture by material-point erosion. International Journal of fracture 184:3-16.
[13] Li, B., Pandolfi, A. and Ortiz, M. 2015. Material-point erosion simulation of dynamic fragmentation of metals. Mechanics of Materials 80:288-297.

[14] Li, B., Habbal, F. and Ortiz, M. 2010. Optimal transportation meshfree approximation schemes for fluid and plastic flows. International Journal for Numerical Methods in Engineering 83:15411579.

[15] Li, B., Kadane, A., Ravichandran, G. and Ortiz, M. 2012. Verification and validation of the optimal-transportation meshfree (OTM) simulation of terminal ballistics. International Journal for Impact Engineering 42:25-36.

[16] S. Mindess and A. Bentur 1985. A preliminary study of the fracture of concrete beams under impact loading, using highspeed photography. Cement and Concrete Research, 15(3):474-484.

[17] S. Mindess, N. Banthia, and C. Yan 1986 The fracture-toughness of concrete under impact loading. Cement and Concrete Research, 17(2):231-241.

[18] Banthia, N., Mindess, S., Bentur, A. and Pigeon, M. 1989. Impact testing of concrete using a drop-weight impact machine. Experimental Mechanics, 29(1):63-69.

[19] Banthia, N., Mindess, S. and Trottier, J. 1996. Impact resistance of steel fiber reinforced concrete. ACI Materials Journal, 93(5):472-479.

[20] Wang, N., Mindess, S. and Ko, K. 1996. Fibre reinforced concrete beams under impact loading. Cement and Concrete Research, 26(3):363-376.

[21] Banthia, N., Yan, C. and Sakai, K. 1998. Impact resistance of concrete plates reinforced with a fiber reinforced plastic grid. ACI Materials Journal, 95(1):11-18. 
[22] Zhang, X.X., Ruiz, G., Yu, R.C. and Tarifa, M. 2009. Fracture behaviour of highstrength concrete at a wide range of loading rates. International Journal of Impact Engineering, 36:1204-1209.

[23] Zhang, X.X., Yu, R.C., Ruiz, G., Tarifa, M. and Camara, M.A. 2010. Effect of loading rate on crack velocities in HSC. International Journal of Impact Engineering, 37:359-370.

[24] Ruiz, G., Zhang, X.X., Yu, R.C., Porras, R., Poveda, E. and del Viso, J.R. 2011. Effect of loading rate on fracture energy of high-strength concrete. Strain, 47(6):518524.
[25] Zhang, X.X., Ruiz, G. and Yu, R.C. 2010. A new drop-weight impact machine for studying fracture processes in structural concrete. Strain, 46(3):252-257.

[26] Arroyo, M. and Ortiz, M. 2006. Local maximum-entropy approximation schemes: a seamless bridge between finite elements and meshfree methods. International Journal for Numerical Methods in Engineering 13:2167-2202,

[27] Navas, P., López-Querol S., Yu R.C. and $\mathrm{Li}$ B. 2015. B-bar based algorithm applied to meshfree numerical schemes to solve unconfined seepage problems through porous media. International Journal for Numerical and Analytical Methods in Geomechanics. DOI:10.1002/nag.2472 